Canadian

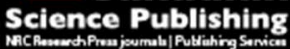

Applied Physiology, Nutrition, and Metabolism Physiologie appliquée, nutrition et métabolisme

\title{
Exercise training and weight loss, not always a happy marriage: single blind exercise trials in females with diverse BMI
}

\begin{tabular}{|r|l|}
\hline Journal: & Applied Physiology, Nutrition, and Metabolism \\
\hline Manuscript ID & apnm-2017-0577.R1 \\
\hline Manuscript Type: & Article \\
\hline Date Submitted by the Author: & 24-Oct-2017 \\
\hline Complete List of Authors: & $\begin{array}{l}\text { Jackson, Matthew ; Liverpool Hope University, School of Health Sciences } \\
\text { Fatahi, Fardin; Bangor University } \\
\text { Alabduljader, Kholoud; Bangor University } \\
\text { Jelleyman, Charlotte ; Leicester General Hospital } \\
\text { Moore, Jonathan; Bangor University } \\
\text { Kubis, Hans-Peter; Bangor University }\end{array}$ \\
\hline $\begin{array}{r}\text { Is the invited manuscript for } \\
\text { consideration in a Special } \\
\text { Issue? : }\end{array}$ & \\
\hline Keyword: & exercise, obesity, body mass maintenance, energy regulation, hormones \\
\hline
\end{tabular}


1 Exercise training and weight loss, not always a happy marriage: single blind exercise trials in

2 females with diverse BMI

3

4 Matthew Jackson ${ }^{1,}{ }^{*}$, ,,$F a r d i n ~ F a t a h i{ }^{2,}{ }^{*}$, Kholoud Alabduljader $^{3}$, Charlotte Jelleyman $^{4}$, Jonathan P.

5 Moore $^{5}$, Hans-Peter Kubis ${ }^{6}$

6 1-6 College of Health \& Behavioural Sciences, Bangor University, Bangor, LL57 2PZ, UK.

$7 \quad *$ These authors contributed equally to this work

8 \# Corresponding author

9 Author current affiliations and contact details:

101 School of Health Sciences, Liverpool Hope University, Hope Park, Liverpool, L17 8TZ, UK; Tel: 0044

11 (0)151 291 3758; Fax: 0044 (0)151 291 3441; E-mail: jacksom1@hope.ac.uk

122 College of Health \& Behavioural Sciences, Bangor University, Bangor, LL57 2PZ, UK; Email:

13 fatahi.fardin@yahoo.com

143 College of Health \& Behavioural Sciences, Bangor University, Bangor, LL57 2PZ, UK; Email:

15 kholoud911@gmail.com

164 Leicester Diabetes Centre, Leicester General Hospital, Gwendolen Road, Leicester, LE5 4PW, UK;

17 Email: cj136@le.ac.uk

185 College of Health \& Behavioural Sciences, Bangor University, Bangor, LL57 2PZ, UK; Email:

19 j.p.moore@bangor.ac.uk

206 College of Health \& Behavioural Sciences, Bangor University, Bangor, LL57 2PZ, UK; Email:

21 pes203@bangor.ac.uk 


\section{Abstract}

24 Individuals show high variability in body weight responses to exercise training. Expectations and 25 motivation towards effects of exercise on body weight might influence eating behaviour and could 26 conceal regulatory mechanisms. We conducted two single-blind exercise trials (4 weeks (study 1 ) and 8 weeks (study 2)) with concealed objectives and exclusion of individuals with weight loss intention. Circuit exercise training programs ( 3 times a week (45-90 min), intensity $50-90 \% \mathrm{VO}_{2}$ peak, for 4 and 8 weeks) were conducted. 34 females finished the 4 weeks intervention and 36 females the 8 weeks intervention. Overweight/obese (OV/OB) and lean (L) female participants' weight/body composition responses were assessed and fasting and postprandial appetite hormone levels (PYY, insulin, amylin, leptin, ghrelin) were measured pre and post intervention for understanding potential contribution to individuals' body weight response to exercise training (study 2). Exercise training in both studies did not lead to a significant reduction of weight/BMI in the participants' groups, however, lean participants gained muscle mass. Appetite hormones levels were significantly $(p<0.05)$ altered in the $O V / O B$ group affecting fasting $(-24 \%)$ and postprandial amylin $(-14 \%)$ levels. Investigation of individuals' BMI responses using multiple regression analysis revealed that levels of fasting leptin, postprandial amylin increase, and BMI were significant predictors of BMI change explaining about $43 \%$ of the variance. In conclusion, tested exercise training did not lead to weight loss in female participants, while a considerable proportion of variance in body weight response to training could be explained by individuals' appetite hormone levels and BMI. 


\section{Introduction}

48 Exercise is often prescribed for weight loss (Donnelly et al. 2009). However, although weight loss is

49 often reported (Ross et al. 2000, 2004), exercise training does not always result in weight loss and

50 often reveals high individual variability in body weight changes (King et al. 2007, 2008, Barwell et al.

51 2009). Possible causes of less than expected weight outcomes are suggested to be modified

52 appetite, perceived reinforcement value of food and altered unstructured physical activity (Blundell

53 et al. 2003, King et al. 2007, Church et al. 2009). Accordingly, the concept of compensators and non-

54 compensators of negative energy balance has been established, although causes for individuals'

55 responses are still debated (Finlayson et al. 2009). Energy balance, and therefore body weight, is

56 regulated by mediators released from gastrointestinal apparatus, pancreas and fat tissue, as well as

57 nutrients. Tonic and phasic signals provide important information about the energy status to the

58 brain. Leptin and insulin, as well as possibly amylin, providing tonic information about energy status;

59 ghrelin, as well as PYY (1-36, 3-36), GLP-1, CCK, and again amylin and insulin providing phasic signals

60 direct to the hypothalamus but also to the hind brain (Suzuki et al. 2010). Moreover, the levels of

61 response to hormonal changes are not restricted to satiety and hunger but expand to neuronal

62 systems connected to hedonic responses, like the mesolimbic dopamine neurons towards leptin and

63 insulin (Figlewicz 2003, Figlewicz and Benoit 2009) or ghrelin's involvement in reward processing

64 (Jerlhag et al. 2007). Alterations on hormonal levels are shown to contribute to the regulation of

65 energy balance if challenged by exercise training (Stensel 2010); with training PYY and ghrelin are more consistently found to be altered then others (Broom et al. 2009, Ueda et al. 2009, Kawano et al. 2013). A possible influencing factor in exercise training studies is the control of motivation and intention of individuals to restrain their food intake, even if this may not be wanted by the experimenters. It is possible that some studies are biased towards weight loss based on the recruitment of participants who may be motivated to lose weight and not naïve towards study aims and objectives. To investigate possible mediators influencing individuals' weight loss response to 
73 the other lasting 8 weeks (study 2). Group-based circuit training exercises were performed at 50-95\%

74 VO2peak, 3 times a week, for 45-90 min. Energy intake was ad libitum in both studies, which were

75 designed with the intention of avoiding the formerly mentioned influence of motivation to lose

76 weight. Thus, aims and objectives were concealed from the participants and spurious objectives

77 were provided. Females over a wide range of BMI were recruited as participants and individuals who expressed an intention to lose weight were excluded. In the first study (4 weeks), a randomized control design was used and body characteristics and composition, as well as cardiovascular fitness were measured pre and post training. In the second study ( 8 weeks), fasting and postprandial blood samples were taken for measurements of appetite hormones and metabolites, body composition, cardiovascular fitness, and resting metabolic rate assessed pre and post intervention. We hypothesized that a) females with overweight/obesity and leanness would regulate their body weight successfully leading to no weight/BMI changes after the training interventions; b) measured appetite hormones levels (PYY, insulin, amylin, leptin, ghrelin) could be used to explain individuals' variance in BMI changes; c) hormone levels would be affected by exercise training leading to reduced levels of appetite suppressing hormones.

\section{Materials and methods}

The two studies were approved by Bangor University ethics committee and the North Wales

Research Ethics Committee - West (Betsi Cadwaladr University Health Board - REC No 11/WA/0321 and 12/WA/0118). All participants were given written and verbal information and participants provided written informed consent. 
97 exercise training group or a control group. In study 2, 56 females were recruited for an 8 weeks

98 training intervention with 36 females completing. For both studies, recruitment was performed

99 using emails to students and employees of Bangor University and posters in the Bangor area. To

100 conceal the aims and objectives of the research, potential participants were informed that the study

101 investigated the influence of exercise training on cognitive performance and cardiovascular fitness. In

102 study 2, an incentive for taking part was the reimbursement for effort with a pair of trainers up to

$103 £ 100$ value. The element of deception in both studies was achieved using a computer based

104 cognitive sorting task for measuring reaction times in recognising combinations of pictures and

105 words. Participant information sheets were written according to the spurious objectives, and any

106 questions arising were answered by researchers accordingly. Participants were debriefed after the

107 intervention.

Potential participants were selected to take part in the study based on their responses to a pre-

screening questionnaire assessing health, physical activity, general diet habits (i.e. restrictive diet).

To avoid participants' bias towards weight loss and potential dieting, we used Aizen's theory of planned behaviour (Ajzen 1991) as a framework for including/excluding participants based on current intention to lose weight (Sørensen et al. 2005). Participants were aged between 18 and 40; $\mathrm{BMI}$ categories were lean $(\mathrm{L})<25 \mathrm{~kg} / \mathrm{m}^{2}$ and overweight/obese $(\mathrm{OV} / \mathrm{OB})>25 \mathrm{~kg} / \mathrm{m}^{2}$; healthy; sedentary; not following any type of specialised diet; and having not stated an intention to lose weight.

Exercise sessions were circuit based (e.g.running on the spot, lunges, star jumps, sit-ups, press-ups and squats) for both studies and completed 3 times a week. Length of sessions was 60 minutes in study 1 , and between $40-90$ minutes in study 2 , dependent upon the intensity of exercise required to achieve equal exercise energy expenditure across two training groups (descriptions follows). All sessions were completed in small training groups (5-10 participants) and supervised by 3 members of the research team. Participants trained in groups according to their BMI group (L or OV/OB). In 
122 study 1, after randomized distribution into control and exercise group, individuals trained on a target

123 heart rate representing $70-80 \%$ of their heart rate at $\dot{V} \mathrm{O}_{2}$ peak. The control group did not take part in

124 any exercise training. In study 2, to include the influence of exercise intensity into the design,

125 participants were randomly assigned to two exercise intensities moderate (50-60\% $\dot{V} \mathrm{O}_{2}$ peak) (L and

$126 \mathrm{OV} / \mathrm{OB}$ ) and high intensity (80-90\% $\dot{V} \mathrm{O}_{2}$ peak) ( $\mathrm{L}$ and OV/OB) training groups. Exercise intensity was

127 used as a continuous variable based on heart rate recordings as well as a covariate in the statistical

128 analysis due to high variability of achieved target heart rates in the groups. Heart rate was

129 continuously recorded throughout all sessions and an approximation of energy expenditure was

130 calculated based on $\dot{V} \mathrm{O}_{2}$ peak assessment. Training intensity was controlled by a telemetric heart

131 rate monitoring system (Activio, Activio Sport System, Sweden) displaying the live heart rates of

132 each participant. HR data were analysed to calculate mean exercise intensity and estimates of

133 energy expenditure throughout the 8 weeks training (study 2 ) to achieve a matched total exercise

134 energy expenditure across groups.

135 Anthropometry

136 Body mass and composition were measured using a beam scale (Seca, Germany) and dual-energy x-

137 ray absorptiometry (DXA; QDR 4500, Hologic, Bedford, MA, USA).

Resting metabolic measurements

In study 2, after 12 hour overnight fast participants, having refrained from exercise for 48 hours,

resting metabolic rate $\left(\mathrm{kcal} \cdot \mathrm{min}^{-1}\right)$ and respiratory exchange ratio $\left(\mathrm{RER} ; \dot{V} \mathrm{CO}_{2} / \dot{V} \mathrm{O}_{2}\right)$ were measured

by indirect calorimetry (Oxycon Pro, Erich Jaegar, Germany) in a supine position for 30 minutes;

heart rate (Polar RS800CX, Polar Electro Oy, Kempele, Finland) was also recorded.

143 Blood sampling and analysis

In study 2 , under both overnight-fasting and postprandial conditions $12 \mathrm{ml}$ of venous blood was 
146 (Accu-Chek ${ }^{\circledast}$ Aviva, Mannheim, Germany). For further measurements, plasma was aliquoted, frozen

147 and stored at $-80^{\circ} \mathrm{C}$. Hormone measurements were carried out by enzyme-linked immunosorbent

148 assay (ELISA) and plate reader (Fluostar Omega, BMG Labtech, Germany). ELISAs were carried out to

149 measure amylin (Millipore, St. Charles, MO, USA) (intra assay CV: 12\% ), insulin (Mercodia, Uppsala,

150 Sweden) (intra assay CV: 7\%), leptin (BioVendor Research and Diagnostic Products, BioVendor -

151 Laboratorni medicina a.s., Czech Republic) (intra assay CV: 7\% ), total ghrelin (Millipore; St. Charles,

152 MO, USA) (intra assay CV: 4\%), and PYY (Millipore Corporation, Billerica, MA, USA) (intra assay CV:

153 12\%). The Homeostasis Model Assessment version 2 (HOMA2) (www.dtu.ox.ac.uk/homacalculator/)

154 was used to calculate beta cell function, insulin resistance and insulin sensitivity. All samples were

155 batch analysed and assayed in duplicate.

156 Test meal

157 In study 2, to analyse potential influence of chronic and phasic appetite hormone changes on

158 individual BMI alterations, participants were given a liquid test meal (Resource ${ }^{\circledR}$ Energy Vanilla

$159200 \mathrm{ml}$, Nestle, Switzerland) following overnight fast according to a modified protocol by Kraemer et

160 al. (2011). The meal provided $300 \mathrm{kcal}$ of which $55 \%$ was carbohydrate, $30 \%$ fat and $15 \%$ protein. This

161 test meal was chosen to avoid variability in intake composition and processing known from more

162 complex meals. Blood samples were taken prior to the test meal at fasting state and precisely 1 hour

163 after consumption. Timing of blood sampling was chosen due to former experiments selecting the

164 time point with the strongest correlation between appetite hormone levels and BMI-based body

165 type. Significant $(\mathrm{p}<0.05)$ correlations between $\mathrm{BMI}$ and appetite hormone levels at fasting $(\mathrm{F})$,

166 postprandial (PP) levels and alterations $(\mathrm{CH})$ were found for insulin $(\mathrm{F}$, rho $=0.49$; $P P$, rho= 0.37 ),

167 amylin $(\mathrm{F}, r h o=0.49 ; \mathrm{CH}$, rho $=-0.48)$, $\mathrm{PYY}(\mathrm{CH}$, rho $=-0.33)$, ghrelin $(\mathrm{CH}$, rho $=0.43)$, leptin $(\mathrm{F}$, rho $=$

168 0.59).

169

Peak oxygen consumption 
170 For both studies, peak oxygen uptake $\left(\dot{V} \mathrm{O}_{2 \text { PEAK }} ; \mathrm{ml} \cdot \mathrm{kg}^{-1} \cdot \mathrm{min}^{-1}\right)$ was measured on a cycle ergometer

171 (Corival 400, Lode, Groningen, Netherlands) using a graded exercise protocol with 1 minute stages

172 (20 watts steps), until exhaustion. Oxygen and carbon dioxide were measured by a metabolic cart

173 (Oxycon Pro, Erich Jaegar, Germany). Heart rate (Polar RS800CX, Polar Electro Oy, Kempele, Finland)

174 and ratings of perceived exertion (Borg 1973) were collected at the end of every stage and at point

175 of exhaustion. $\dot{V} \mathrm{O}_{2 \mathrm{PEAK}}$ was achieved when one of three criteria was met: RER greater than 1.1, RPE

176 of 20 or cycling cadence less than 60rpm. Control subjects in study 1 were not tested for $\dot{V} \mathrm{O}_{2 \text { PEAK. }}$

177 Statistical analysis

178 All statistical analyses were performed using IBM SPSS Statistics 20. Data were analysed either by

179 one-way ANOVA (baseline characteristics), ANCOVA using exercise intensity as a covariate or by

180 mixed model ANOVA and appropriate post hoc analysis, after assumptions had been met and

181 outliers removed. Pearson's and Spearman's rho correlations were used to analyse relationships

182 between variables. Multiple regression analyses using the enter and backward methods were

183 performed on variables of interest. All data are reported as means and \pm standard deviation.

184 Statistical significance was set at $p<0.05$.

Results

In the first study, 34 female participants of the 40 recruited finished the intervention. Mixed model

ANOVA with repeated measures revealed that there were no significant alterations in weight/BMI

after the 4 weeks, neither in the exercise training group nor in the non-exercising control group

(Table 1). Consequently, exercise related energy expenditure was compensated and body

weight/BMI was maintained. Further analysis of body composition showed that there was a 
trial (control/exercise) $(p=0.041)$, and interaction of time $x$ baseline body fat [\%] $(p=0.046))$, (Table

1). Moreover, an increase in lean mass ( $\mathrm{kg}$ ) was significant only in the lean participants of the

exercise group, who gained about $1 \mathrm{~kg}$ lean mass (increase of lean mass over time $(p=0.009)$,

interaction of time $x$ baseline fat percentage [\%] $(p=0.028)$ and time $x$ trial (control/exercise)

$(p=0.05))$, (Table 1). Individual alterations in body characteristics over the 4 weeks intervention

period are depicted in Figure 1; positive effects on body composition were restricted to lean

participants of the exercise group but without alteration of weight/BMI.

The second study used principally the same experimental design but omitting a non-exercising

group; the training program was performed for 8 weeks. Additionally, exercise energy expenditure

was matched across participants using a wider range of training intensities (50-90\%VO2peak).

Training intensity was implemented as a covariate to investigate its possible influence on weight and

body composition. This was suggested based on outcomes of study 1 where body composition changes were restricted to lean participants who trained on higher absolute intensity. Moreover, fasting and postprandial blood samples were collected for the analysis of appetite hormones and metabolites pre and post intervention. The design was chosen to confirm outcomes of study 1 with a further focus on the investigation of underlying factors responsible for individual weight/BMI responses to exercise in lean and overweight/obese females.

From the 56 recruited females for the 8 weeks training program, 36 females finished the study.

212 Baseline body characteristics and blood parameters of the participants are given in Table 2. OV/OB

213 participants had higher $(\mathrm{p}<0.05)$ levels of $\mathrm{BMI}$, weight, fat mass, lean mass, and RMR as well as

214 lower relative $\mathrm{VO}_{2}$ peak compared with $\mathrm{L}$ individuals (Table 2).

Training compliance was $\sim 85 \%$ across the training groups with no difference between groups; heart rate based estimates of total exercise energy expenditure, amounting to $\sim 3400 \mathrm{kcal}$ after 8 weeks,

217 and was matched across the groups (Table 3). Moreover, mean training intensity in percent heart rate reserve was about $65 \%$ with no difference between lean and OV/OB groups (Table 3 ). 
219 Mixed model ANOVA with body type (BMI groups) as between factor and training intensity as 220 covariate revealed that 8 weeks training did not lead to significant alterations of BMI/weight in either group (no significant main effect of time, or interactions of time x group) (Table 3); hence, both groups compensated the exercise energy expenditure over the training period confirming outcomes of study 1 . In terms of body composition changes, females of the lean group lost body fat while participants of the OV/OB group remained unaltered after the training period (no significant time effect was reported for body fat [\%] change but a significant $(p=0.008)$ interaction of time $x$ body type). Moreover, lean mass ( $\mathrm{kg}$ ) was not affected (non-significant time effect) but there was a significant interaction time $x$ training intensity $(p=0.025)$ supporting the hypothesis that lean mass changes have been influenced by training intensity (Table 3). A further splitting of the data (Table 4) in moderate and high intensity training groups without consideration of BMI shows that the higher intensity group tended to gain more lean mass than the moderate exercise group. Moreover, a significant correlation $(\mathrm{R}=0.458 ; \mathrm{p}=0.006)$ between training intensity and $\dot{V} \mathrm{O}_{2 \text { PEAK }}$ showed that individuals with higher cardiovascular fitness tended to train harder.

In summary, the second study confirmed that lean and OV/OB females compensate exercise induced energy expenditure without losing weight but positive body composition changes were more apparent in lean participants being possibly related to training intensity.

To further investigate individuals' weight/BMI response to training (individuals' post intervention changes in BMI and body composition are shown in Figure 2), we analysed fasting and postprandial blood samples.

ANOVA analysis of pre intervention levels of the two groups revealed significant differences in fasting levels for leptin, PYY, insulin, and amylin between groups (Table 5). Moreover, postprandial increases in amylin and PYY were significantly different between L and OV/OB groups (Table 5). After

2428 weeks exercise training, fasting and postprandial levels of amylin were significantly reduced in the 243 OV/OB group but not in the L group (no significant main effect of time, significant interaction of time 
$244 x$ body type, $p<0.001$ for fasting and postprandial levels, $p=0.004$ ) (Table 5). The postprandial

245 increase of amylin, which was significantly different between groups, was unaltered after the

246 training revealing an unchanged higher increase of amylin after the test meal in L group females

247 compared with females of the OV/OB group (Table 5). Multiple regression analysis showed that

248 postprandial amylin levels after the intervention were determined $\left(R^{2}=0.34, p=0.002, n=33\right)$ by

249 fasting glucose levels $(\beta=0.35, p=0.027)$ and postprandial increase in glucose $(\beta=0.50, p=0.002)$.

250 Fasting and postprandial levels of insulin, leptin, PYY, total ghrelin were unchanged after exercise

251 training (no significant main effect and interactions) (Table 5).

252 To further associate hormonal levels with individuals' BMI response to exercise (see also figure 2),

253 we performed multiple regression analysis (enter method) using appetite hormone levels as

254 predictor variables and body characteristics for post intervention BMI changes. Analysis led to a

255 significant model for the BMI change of participants who finished the training; the model used post

256 intervention levels of leptin $(\beta=0.59, p=0.002)$ and postprandial amylin change $(\beta=-0.37, p=0.03)$, and

257 pre-intervention $\mathrm{BMI}(\beta=-0.44, p=0.02)$ as predictor variables. The three variables explain $43 \%$ of the

258 variance of the $\mathrm{BMI}$ alterations $\left(\mathrm{R}^{2}=0.43, \mathrm{p}=0.002, \mathrm{n}=30\right)$ after training. Other hormone parameters

259 did not lead to significant model improvements.

Metabolic alterations

There were significantly higher levels in insulin sensitivity, beta cell function and lower insulin

resistance in the L- than in the OV/OB group. However, comparisons of HOMA 2 parameters 
267 We conducted two exercise training interventions with sedentary females with concealed aims and

268 objectives of the study and excluding participants who expressed an intention to lose weight. To our

269 knowledge, this is the first exercise training study which tried to achieve ad libitum conditions for

270 participants whilst avoiding the influence of explicit motivation towards weight loss. Both

271 interventions did not lead to significant weight loss/BMI change in both OV/OB and L groups after 4

272 and 8 weeks training. This finding is consistent with our first hypothesis and we interpret this as

273 indicative of intact weight regulation over the periods of the exercise training, even in females with

274 high BMI (e.g. overweight/obesity). This outcome, considering the mean weight changes, as well as

275 individual weight responses to exercise, is dissimilar to results published earlier (King et al. 2007,

276 2008). For example, King et al. (2008) reported considerable weight loss with high variability

277 amongst overweight/obese participants and outcomes were skewed towards weight loss. This

278 suggests that BMI/weight alterations in comparable training studies might be partially driven by

279 participants' intention to lose weight with concomitant consequences for eating behaviour rather

280 than a singular effect of exercise. Additionally, recent work showed that the window for a

281 satisfactory increase in total energy expenditure is narrow; in a large, diverse population sample it

282 was shown that only about 7-9\% of the variance in total energy expenditure was explained by

283 physical activity (Pontzer et al. 2016). These authors assume that homeostatic regulation not only

284 affects weight but also total energy expenditure.

285 Our study results on group level (i.e. no weight change over time), though, do not explain the

286 individuals' weight response to training which varied strongly from considerable weight loss to

287 weight gain, a consistent finding in studies which lead to the concept of compensators and non-

288 compensators (King et al. 2008). As mentioned before, body weight is influenced by homeostatic and

289 hedonic mechanisms, with some authors suggesting that humans are more prone to be driven by

290 hedonic regulation (Berthoud 2011). Indeed, exercise energy depletion could increase the incentive

291 salience of food, like it is known from fasting (Berthoud 2011) and increasing hunger levels have

292 previously been reported following exercise training (King et al. 2009). However, it was suggested 
that alterations in food reward after exercise bouts are not influenced by exercise training and the reward response seems to be more trait-like. Finlayson et al. (2011) did not find alterations in wanting and liking of foods after 12 weeks training but participants who lost weight (responders) had a lesser increase in food reward after a bout of exercise than participants who reduced weight less than predicted (non-responders). Additionally, in our study, participants, not having the objective to lose weight, could have responded to the exhaustion and sensation of effort related to exercise in a self-rewarding manner with the selection of high palatable foods. Furthermore, poor judgement of caloric expenditure could have reduced existing diet restrain. Clearly, these possible factors might have contributed to the variance in the weight outcomes in our study. However, due to our study design we were not able to collect data about any alterations in food reward. On the other hand, it is known that both regulatory processes are heavily interlinked and difficult to separate; in particular appetite hormones are repeatedly shown to influence 'liking' and 'wanting' or reward perception, as well as influencing energy intake and energy metabolism (Volkow et al. 2011). While we gathered no information about the individuals' motives of eating in our study, we still gathered information about appetite hormones responses at fasting and postprandial levels to analyse their possible contribution to the variability of weight/BMI changes after the exercise training intervention. Post intervention, most of the tested appetite hormones revealed no alterations in both groups maintaining the differences detected at baseline. Nonetheless, we found significant alterations of amylin at fasting and postprandial levels in the OV/OB group, while the $\mathrm{L}$ group revealed no changes after the 8 weeks training intervention. Reports about alterations in amylin levels in response to exercise training are sparse; Izadpanah et al. (Izadpanah et al. 2012) and Roberts at al. (2013) reported a reduction of amylin in response to a combined diet exercise intervention in children with obesity for 14 days. Additionally, acute responses to exercise bouts with a reduction of amylin after prolonged exercise bouts (Kraemer et al. 2011) and increase in higher intensity bouts (Kraemer et al. 2002) were recently shown. Mechanistically, amylin expression in beta cells was recently shown to respond directly to glucose availability via carbohydrate- 
response-element-binding-protein (ChREBP) and thioredoxin-interacting-protein (TXNIP) (Jing et al.

320 2014). Indeed, our data revealed that amylin levels were significantly influenced by fasting levels and

321 postprandial increase of glucose supporting this possible connection between amylin levels and

322 altered glucose availability. Moreover, a positive associations between postprandial amylin levels

323 and fasting glucose levels at post intervention was particular strong $(r=0.625, p=0.02)$ in $O V / O B$

group which highlights a possible connection between glucose availability and amylin levels. Clearly,

fasting glucose levels can be influenced via sugar/carbohydrate intake (Sartor et al. 2013) as well as

exercise (Sartor et al. 2010). Theoretically, a stronger depletion in glycogen storage during exercise

in OV/OB individuals who might have more preferred carbohydrate utilization during exercise could

have reduced glucose availability and could have led to reduced amylin levels with consequences for appetite and possible compensatory food intake.

Participation in exercise is often driven by a desire to lose weight (Teixeira et al. 2012). However, individual physiological differences may confound attempts to lose weight. In our study, observed amylin alterations contributed to the individual weight outcomes after the intervention. Indeed, our multiple regression analysis showed that hormone levels of leptin and postprandial amylin increase were best predictors for BMI changes (about $43 \%$ of BMI change variance explained). Leptin is known to be the most important tonic signal of fatness and mainly sensed in the hypothalamus for the intrinsic drive to eat and consequently for the regulation of body weight and energy expenditure

337 (Blundell and Gillett 2001); therefore the contribution of leptin levels in the model is not unexpected. Additionally, leptin's links towards perceptual response to food was recently established identifying fasting leptin levels as a determinant of food reward (Hopkins et al. 2014). However, the strong contribution of amylin for the model is noteworthy. Amylin is known to play a role as a satiogenic signal, inhibits gastric emptying, and possesses glucoregulatory functions; agonists are well established in supporting weight loss in people with obesity (Smith et al. 2007). Moreover, amylin and leptin are shown to share important functions in the hindbrain and 
of leptin responsiveness threshold (Trevaskis et al. 2010). Decreased amylin levels (postprandial and fasting) could increase leptin responsiveness threshold and could have led to increased energy intake in response to exercise training. Consequently, participants who displayed a combination of high levels of leptin and low postprandial increase in amylin were more prone to weight gain during exercise training. However, further work needs to support this interpretation.

Our work has several limitation; firstly, the selection of appetite hormones measured in this study does not exclude the importance and possible contribution of other hormones to the weight response in our study. Clearly, other hormones are consistently shown to be affected by training. Exercise training type, intensity, and duration are certainly factors that could influence outcomes in studies; besides the involvement of restricted dieting. Moreover, knowledge about altered food preference over the training period in terms of caloric density, macronutrient amounts and composition would have supported the interpretation of results largely. However, the need for not disclosing objectives of our study excluded the recording of precise food diaries and assessments of food liking, wanting and preference. However, our study used an ecological training programme which includes exercises and intensities commonly used in leisure centres or gyms. Finally, we used females only; consequently, we can't extrapolate findings towards males. In summary, we have shown that under ad libitum condition 4 and 8 weeks exercise training did not result in weight loss in females over a wide range of BMI. Appetite hormone responses revealed decrease in amylin at fasting and postprandial levels, however this was restricted to overweight/obese participants. A large proportion of variance in BMI changes after training could be explained by postprandial amylin increase and leptin levels, pointing towards an important influence of amylin for weight regulation during exercise training.

\section{Perspective} face less than expected weight loss or even weight gain over an exercise training period. Clearly, 
370 unrealistic expectations about the response of an individual to exercise training impairs exercise

371 participation, in particular in population groups who could largely benefit on many other health

372 levels other than weight loss. In our single blind exercise training study, excluding participants with

373 weight loss intentions, females within a wide range of BMI, did not lose weight on group levels.

374 However, individual weight gains or losses could be explained by appetite hormone levels. In

375 particular, levels of amylin and leptin could explain a significant proportion (43\%) of the variance in

376 BMI changes post training. Our results highlight the need for individualized interventions tailored

377 also to the physiological and not only to psychological characteristics of clients in weight loss

378 programs.

\section{Acknowledgments}

We thank David King, Robert Davies, and Lewis Fox for support in the data collection and training.

We thank David Alan Markland for providing the cognitive sorting task.

\section{Conflict of interest}

The authors declare no conflict of interest.

\section{References}

Ajzen, I. 1991. The theory of planned behavior. Organ. Behav. Hum. Decis. Process, 50(2): 179-211. doi:10.1016/0749-5978(91)90020-T.

Barwell, N.D., Malkova, D., Leggate, M., and Gill, J.M. 2009. Individual responsiveness to exercise1320-1328. doi:10.1016/j.metabol.2009.04.016; 10.1016/j.metabol.2009.04.016. 
393

394

395

396

397

398

399

400

401

402

403

404

405

406

407

408

409

410

411

412

413

414

415

Berthoud, H.-R. 2011. Metabolic and hedonic drives in the neural control of appetite: who is the boss? Curr. Opin. Neurobiol. 21(6): 888-96. doi:10.1016/j.conb.2011.09.004.

Blundell, J.E., and Gillett, A. 2001. Control of food intake in the obese. Obes. Res. 9 Suppl 4: 263S270S. doi:10.1038/oby.2001.129.

Blundell, J.E., Stubbs, R.J., Hughes, D.A., Whybrow, S., and King, N.A. 2003. Cross talk between physical activity and appetite control: does physical activity stimulate appetite? Proc. Nutr. Soc. 62(3): 651-61. doi:10.1079/PNS2003286.

Borg, G.A. 1973. Perceived exertion: a note on history and methods. Med. Sci. Sports, 5(2): 90-3. Available from http://www.ncbi.nlm.nih.gov/pubmed/4721012 [accessed 14 June 2016].

Broom, D.R., Batterham, R.L., King, J.A., and Stensel, D.J. 2009. Influence of resistance and aerobic exercise on hunger, circulating levels of acylated ghrelin, and peptide YY in healthy males. Am. J. Physiol. Integr. Comp. Physiol. 296(1): R29-35. doi:10.1152/ajpregu.90706.2008; 10.1152/ajpregu.90706.2008.

Church, T.S., Martin, C.K., Thompson, A.M., Earnest, C.P., Mikus, C.R., and Blair, S.N. 2009. Changes in weight, waist circumference and compensatory responses with different doses of exercise among sedentary, overweight postmenopausal women. PLoS One, 4(2): e4515. doi:10.1371/journal.pone.0004515.

Donnelly, J.E., Blair, S.N., Jakicic, J.M., Manore, M.M., Rankin, J.W., Smith, B.K., et al. 2009. American College of Sports Medicine Position Stand. Appropriate physical activity intervention strategies for weight loss and prevention of weight regain for adults. Med. Sci. Sports Exerc. 41(2): 45971. doi:10.1249/MSS.0b013e3181949333.

Figlewicz, D.P. 2003. Adiposity signals and food reward: expanding the CNS roles of insulin and leptin. Am. J. Physiol. Regul. Integr. Comp. Physiol. 284(4): R882-92. 
doi:10.1152/ajpregu.00602.2002.

417

418

419

420

421

422

423

424

425

426

427

428

429

430

431

432

433

434

435

436

437

438

Figlewicz, D.P., and Benoit, S.C. 2009. Insulin, leptin, and food reward: update 2008. Am. J. Physiol. Regul. Integr. Comp. Physiol. 296(1): R9-R19. doi:10.1152/ajpregu.90725.2008.

Finlayson, G., Bryant, E., Blundell, J.E., and King, N.A. 2009. Acute compensatory eating following exercise is associated with implicit hedonic wanting for food. Physiol. Behav. 97(1): 62-67. doi:10.1016/j.physbeh.2009.02.002; 10.1016/j.physbeh.2009.02.002.

Finlayson, G., Caudwell, P., Gibbons, C., Hopkins, M., King, N., and Blundell, J. 2011. Low fat loss response after medium-term supervised exercise in obese is associated with exercise-induced increase in food reward. J. Obes. 2011. doi:10.1155/2011/615624.

Hopkins, M., Gibbons, C., Caudwell, P., Hellström, P.M., Näslund, E., King, N.A., et al. 2014. The adaptive metabolic response to exercise-induced weight loss influences both energy expenditure and energy intake. Eur. J. Clin. Nutr. 68(5): 581-6. doi:10.1038/ejcn.2013.277.

Izadpanah, A., Barnard, R.J., Almeda, A.J., Baldwin, G.C., Bridges, S.A., Shellman, E.R., et al. 2012. A short-term diet and exercise intervention ameliorates inflammation and markers of metabolic health in overweight/obese children. Am. J. Physiol. Metab. 303(4): E54250.doi:10.1152/ajpendo.00190.2012; 10.1152/ajpendo.00190.2012.

Jerlhag, E., Egecioglu, E., Dickson, S.L., Douhan, A., Svensson, L., and Engel, J.A. 2007. Ghrelin administration into tegmental areas stimulates locomotor activity and increases extracellular concentration of dopamine in the nucleus accumbens. Addict. Biol. 12(1): 6-16. doi:10.1111/j.1369-1600.2006.00041.x.

Jing, G., Westwell-Roper, C., Chen, J., Xu, G., Verchere, C.B., and Shalev, A. 2014. Thioredoxininteracting protein promotes islet amyloid polypeptide expression through miR-124a and FoxA2. J. Biol. Chem. 289(17): 11807-15. doi:10.1074/jbc.M113.525022. 
439

440

441

442

443

444

445

446

447

448

449

450

451

452

453

454

455

456

457

458

459

460

461

462

Kawano, H., Mineta, M., Asaka, M., Miyashita, M., Numao, S., Gando, Y., et al. 2013. Effects of different modes of exercise on appetite and appetite-regulating hormones. Appetite, 66: 2633. doi:10.1016/j.appet.2013.01.017.

King, N.A., Caudwell, P., Hopkins, M., Byrne, N.M., Colley, R., Hills, A.P., et al. 2007. Metabolic and behavioral compensatory responses to exercise interventions: barriers to weight loss. Obesity (Silver Spring), 15(6): 1373-1383. doi:10.1038/oby.2007.164.

King, N.A., Caudwell, P.P., Hopkins, M., Stubbs, J.R., Naslund, E., and Blundell, J.E. 2009. Dual-process action of exercise on appetite control: increase in orexigenic drive but improvement in mealinduced satiety. Am. J. Clin. Nutr. 90(4): 921-927. doi:10.3945/ajcn.2009.27706; 10.3945/ajcn.2009.27706.

King, N.A., Hopkins, M., Caudwell, P., Stubbs, R.J., and Blundell, J.E. 2008. Individual variability following 12 weeks of supervised exercise: identification and characterization of compensation for exercise-induced weight loss. Int. J. Obes. (Lond). 32(1): 177-184. doi:10.1038/sj.ijo.0803712.

Kraemer, R.R., Acevedo, E.O., Synovitz, L.B., Durand, R.J., Johnson, L.G., Petrella, E., et al. 2002. Glucoregulatory endocrine responses to intermittent exercise of different intensities: plasma changes in a pancreatic beta-cell peptide, amylin. Metabolism, 51(5): 657-63. Available from http://www.ncbi.nlm.nih.gov/pubmed/11979402 [accessed 14 June 2016].

Kraemer, R.R., Francois, M.R., Sehgal, K., Sirikul, B., Valverde, R.A., and Castracane, V.D. 2011. Amylin and selective glucoregulatory peptide alterations during prolonged exercise. Med. Sci. Sports Exerc. 43(8): 1451-1456. doi:10.1249/MSS.0b013e3182114ab9; 10.1249/MSS.0b013e3182114ab9.

Pontzer, H., Durazo-Arvizu, R., Dugas, L.R., Plange-Rhule, J., Bovet, P., Forrester, T.E., et al. 2016. Constrained Total Energy Expenditure and Metabolic Adaptation to Physical Activity in Adult 

Humans. Curr. Biol. 26(3): 410-417. doi:10.1016/j.cub.2015.12.046.

464

Roberts, C.K., Izadpanah, A., Angadi, S.S., and Barnard, R.J. 2013. Effects of an intensive short-term diet and exercise intervention: comparison between normal-weight and obese children. Am. J. Physiol. Regul. Integr. Comp. Physiol. 305(5): R552-7. doi:10.1152/ajpregu.00131.2013.

Ross, R., Dagnone, D., Jones, P.J., Smith, H., Paddags, A., Hudson, R., et al. 2000. Reduction in obesity and related comorbid conditions after diet-induced weight loss or exercise-induced weight loss in men. A randomized, controlled trial. Ann. Intern. Med. 133(2): 92-103. Available from http://www.ncbi.nlm.nih.gov/pubmed/10896648 [accessed 14 June 2016].

Ross, R., Janssen, I., Dawson, J., Kungl, A.-M., Kuk, J.L., Wong, S.L., et al. 2004. Exercise-induced reduction in obesity and insulin resistance in women: a randomized controlled trial. Obes. Res. 12(5): 789-98. doi:10.1038/oby.2004.95.

Sartor, F., Jackson, M.J., Squillace, C., Shepherd, A., Moore, J.P., Ayer, D.E., et al. 2013. Adaptive metabolic response to 4 weeks of sugar-sweetened beverage consumption in healthy, lightly active individuals and chronic high glucose availability in primary human myotubes. Eur. J. Nutr. 52(3): 937-948. doi:10.1007/s00394-012-0401-x; 10.1007/s00394-012-0401-x.

Sartor, F., de Morree, H.M., Matschke, V., Marcora, S.M., Milousis, A., Thom, J.M., et al. 2010. Highintensity exercise and carbohydrate-reduced energy-restricted diet in obese individuals. Eur. J. Appl. Physiol. 110(5): 893-903. Sdoi:10.1007/s00421-010-1571-y.

Smith, S.R., Blundell, J.E., Burns, C., Ellero, C., Schroeder, B.E., Kesty, N.C., et al. 2007. Pramlintide treatment reduces 24-h caloric intake and meal sizes and improves control of eating in obese subjects: a 6-wk translational research study. Am. J. Physiol. Metab. 293(2): E620-7. doi:10.1152/ajpendo.00217.2007. 

changes, and 18-y mortality in overweight individuals without co-morbidities. PLoS Med. 2(6):

Stensel, D. 2010. Exercise, appetite and appetite-regulating hormones: implications for food intake and weight control. Ann. Nutr. Metab. 57 Suppl 2: 36-42. doi:10.1159/000322702. 
508 Table 1: Participants characteristic pre and post 4 weeks exercise training (study 1)

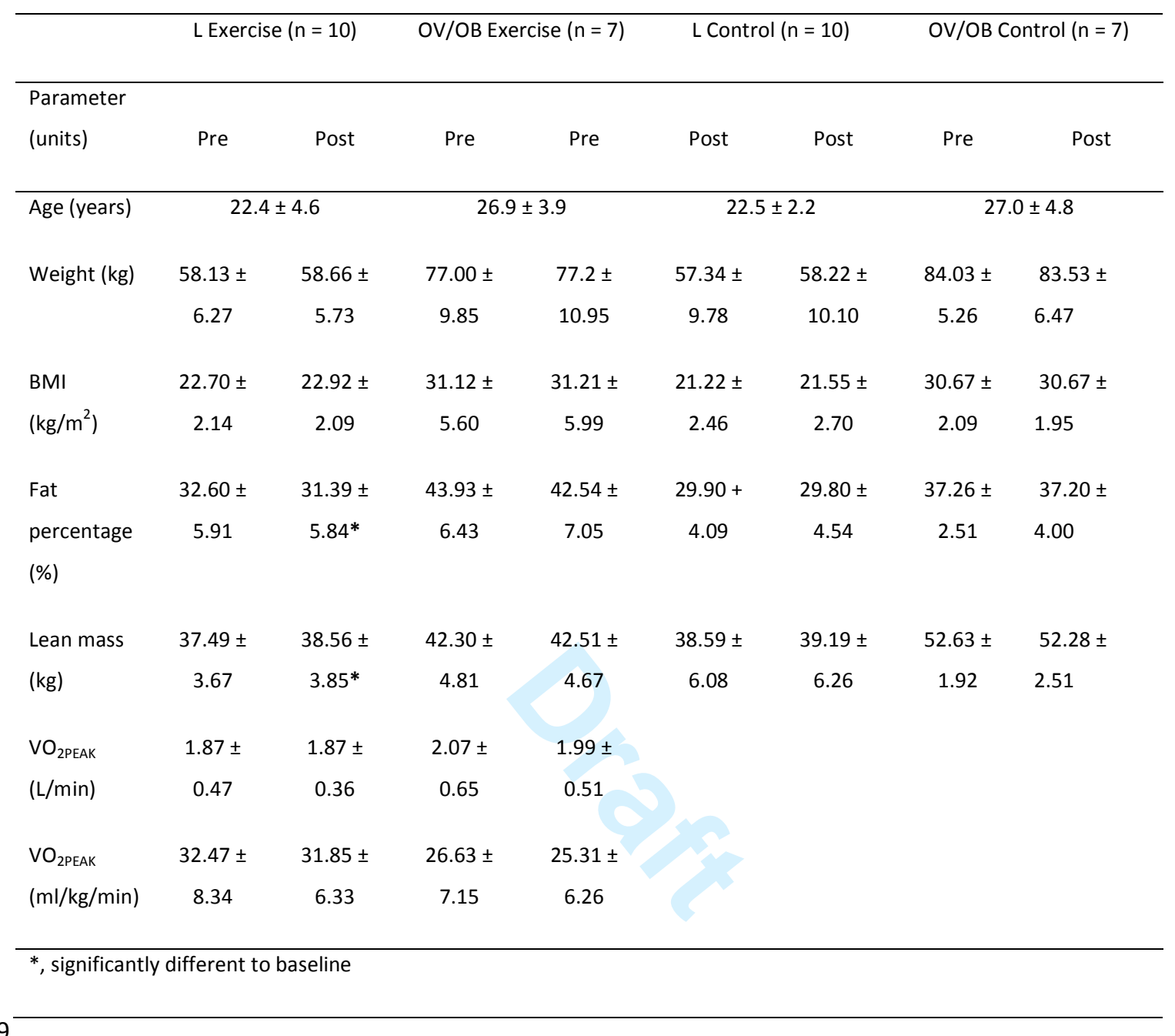

509

510

511

512

513

514

515

516 
517 Table 2: Anthropometric and metabolic parameters of participants at baseline (Study 2)

\begin{tabular}{|c|c|c|}
\hline Parameters (units) & OV/OB $(n=23)$ & $L(n=11)$ \\
\hline Age (years) & $23.39 \pm 5.70$ & $24.55 \pm 6.93$ \\
\hline BMI $\left(\mathrm{kg} \mathrm{m}^{-2}\right)$ & $30.27 \pm 3.66 *$ & $22.41 \pm 2.14$ \\
\hline Weight (kg) & $82.78 \pm 11.88 *$ & $63.71 \pm 5.60$ \\
\hline Fat mass (\%) & $38.74 \pm 4.88 *$ & $29.32 \pm 4.72$ \\
\hline Lean mass ( $\mathrm{kg}$ ) & $49.13 \pm 5.44 *$ & $43.32 \pm 3.99$ \\
\hline Fasting glucose $\left(\mathrm{mmol} \mathrm{I}^{-1}\right)$ & $4.56 \pm 0.42$ & $4.51 \pm 0.44$ \\
\hline Total cholesterol $\left(\mathrm{mmoll}^{-1}\right)$ & $3.85 \pm 0.83$ & $3.59 \pm 0.56$ \\
\hline $\mathrm{HDL}\left(\mathrm{mmol} \mathrm{I}^{-1}\right)$ & $1.51 \pm 0.47$ & $1.68 \pm 0.41$ \\
\hline $\mathrm{LDL}\left(\mathrm{mmol} \mathrm{I}^{-1}\right)$ & $2.24 \pm 0.71$ & $1.75 \pm 0.63$ \\
\hline $\mathrm{TG}\left(\mathrm{mmoll}^{-1}\right)$ & $1.06 \pm 0.37$ & $0.80 \pm 0.00$ \\
\hline $\mathrm{VO}_{2}$ peak $\left(I \mathrm{~min}^{-1}\right)$ & $2.64 \pm 0.49$ & $2.92 \pm 0.56$ \\
\hline $\mathrm{VO}_{2}$ peak $\left(I \mathrm{~min}^{-1} \mathrm{~kg}^{-1}\right)$ & $32.58 \pm 6.27 *$ & $45.89 \pm 0.85$ \\
\hline $\operatorname{RMR}\left(\mathrm{kcal} \mathrm{d}^{-1}\right)$ & $1619.2 \pm 318.9 *$ & $1361.4 \pm 178.9$ \\
\hline RER & $0.77 \pm 0.06$ & $0.79 \pm 0.07$ \\
\hline $\begin{array}{l}* \text { significant group diffe } \\
\text { Lipoprotein, LDL; Triglycer }\end{array}$ & $\begin{array}{l}\text { 5; High Density Lipc } \\
\text { ting Metabolic Rat } \\
\text { Ratio, RER }\end{array}$ & $\begin{array}{l}\text { L; Low Density } \\
\text { piratory Exchange }\end{array}$ \\
\hline
\end{tabular}

518

519

520

521

522

523

524

525

526

527

528 
529 Table 3: Training parameters and alterations of anthropometric and metabolic characteristics of 8

530 weeks training study

\begin{tabular}{|c|c|c|}
\hline Parameter (units) & $O V / O B(n=23)$ & $L(n=11)$ \\
\hline Training Energy Expenditure (kcal) & $3324.7 \pm 1060.4$ & $3194.2 \pm 1344.0$ \\
\hline Training intensity (Watt) & $89.94 \pm 36.13$ & $78.84 \pm 20.06$ \\
\hline Training intensity (\% Heart Rate Reserve) & $60.47 \pm 11.09$ & $65.10 \pm 11.98$ \\
\hline$\Delta \mathrm{BMI}\left(\mathrm{kg} \mathrm{m}^{-2}\right)$ & $0.15 \pm 0.48$ & $0.02 \pm 0.33$ \\
\hline$\Delta$ Weight (kg) & $0.43 \pm 1.69$ & $0.08 \pm 0.96$ \\
\hline$\Delta$ Fat mass $(\%)$ & $0.15 \pm 1.43$ & $-1.16 \pm 1.12 \#$ \\
\hline$\Delta$ Lean mass $(\mathrm{kg})$ & $0.15 \pm 1.23+$ & $0.61 \pm 1.18+$ \\
\hline$\Delta$ Fasting glucose $\left(\mathrm{mmol}^{-1}\right)$ & $0.08 \pm 0.45$ & $0.28 \pm 0.40$ \\
\hline$\Delta$ Total cholesterol $\left(\mathrm{mmol} \mathrm{I}^{-1}\right)$ & $0.27 \pm 0.64$ & $0.23 \pm 0.60$ \\
\hline$\Delta \mathrm{HDL}\left(\mathrm{mmol} \mathrm{I}^{-1}\right)$ & $-0.10 \pm 0.23$ & $0.05 \pm 0.35$ \\
\hline$\Delta \mathrm{LDL}\left(\mathrm{mmol} \mathrm{I}^{-1}\right)$ & $0.25 \pm 0.48$ & $0.17 \pm 0.50$ \\
\hline$\Delta \mathrm{TG}\left(\mathrm{mmol} \mathrm{I}^{-1}\right)$ & $0.12 \pm 0.26$ & $0.05 \pm 0.18$ \\
\hline$\Delta$ VO2peak $\left(\mathrm{I} \mathrm{min}^{-1}\right)$ & $0.05 \pm 0.41$ & $-0.23 \pm 0.32$ \\
\hline$\Delta$ VO2peak $\left(I \mathrm{~min}^{-1} \mathrm{~kg}^{-1}\right)$ & $0.61 \pm 5.10$ & $-3.43 \pm 4.74$ \\
\hline$\Delta \mathrm{RMR}\left(\mathrm{kcal} \mathrm{d}^{-1}\right)$ & $44.86 \pm 250.95$ & $116.57 \pm 182.85$ \\
\hline$\triangle \mathrm{RER}$ & $0.032 \pm 0.08$ & $0.024 \pm 0.11$ \\
\hline
\end{tabular}

531

532

533

534

535

536

537

538 
539 Table 4: Alterations in anthropometric and metabolic parameters after 8 weeks moderate and high

$540 \quad$ intensity exercise training

\begin{tabular}{|c|c|c|c|c|}
\hline Parameters (units) & $\begin{array}{l}\text { Moderate Intensity } \\
(n=16)\end{array}$ & $\begin{array}{l}\text { Change (post -pre } \\
\text { intervention } \\
\text { levels) }\end{array}$ & High Intensity $(n=18)$ & $\begin{array}{l}\text { Change (post -pre } \\
\text { intervention } \\
\text { levels) }\end{array}$ \\
\hline Age (years) & $23.06 \pm 5.27$ & & $24.35 \pm 6.74$ & \\
\hline BMI $\left(\mathrm{kg} \mathrm{m}^{-2}\right)$ & $27.14 \pm 4.75$ & $0.11 \pm 0.61$ & $28.11 \pm 5.17$ & $0.01 \pm 0.62$ \\
\hline Weight (kg) & $74.13 \pm 11.85$ & $0.33 \pm 1.64$ & $78.83 \pm 14.50$ & $0.02 \pm 1.76$ \\
\hline Fat mass (\%) & $35.39 \pm 6.44$ & $0.04 \pm 1.34$ & $35.84 \pm 6.84$ & $-0.81 \pm 1.74$ \\
\hline Lean mass (kg) & $45.84 \pm 5.14$ & $-0.01 \pm 1.14$ & $48.39 \pm 6.50$ & $0.55 \pm 1.26$ \\
\hline Fasting glucose $\left(\mathrm{mmol} \mathrm{I}^{-1}\right)$ & $4.37 \pm 0.39$ & $0.30 \pm 0.46 \dagger$ & $4.75 \pm 0.36$ & $-0.02 \pm 0.34 \dagger$ \\
\hline $\begin{array}{l}\text { Fasting cholesterol (mmol l- } \\
{ }^{1} \text { ) }\end{array}$ & $3.81 \pm 0.76$ & $0.31 \pm 0.74$ & $3.71 \pm 0.76$ & $0.15 \pm 0.49$ \\
\hline $\mathrm{HDL}\left(\mathrm{mmol} \mathrm{l}^{-1}\right)$ & $1.72 \pm 0.44$ & $-0.14 \pm 0.30$ & $1.40 \pm 0.40$ & $0.03 \pm 0.22$ \\
\hline $\operatorname{LDL}\left(\mathrm{mmoll}^{-1}\right)$ & $2.02 \pm 0.61$ & $0.30 \pm 0.54$ & $2.10 \pm 0.80$ & $0.10 \pm 0.43$ \\
\hline $\mathrm{TG}\left(\mathrm{mmol} \mathrm{I}^{-1}\right)$ & $0.90 \pm 0.18$ & $0.12 \pm 0.26$ & $1.04 \pm 0.41$ & $0.08 \pm 0.24$ \\
\hline $\mathrm{VO}_{2}$ peak $\left(I \mathrm{~min}^{-1}\right)$ & $2.75 \pm 0.53$ & $-.0 .84 \pm 0.47$ & $2.72 \pm 0.52$ & $0.02 \pm 0.32$ \\
\hline $\mathrm{VO}_{2}$ peak $\left(\mathrm{min}^{-1} \mathrm{~kg}^{-1}\right)$ & $38.06 \pm 8.39$ & $-1.18 \pm 5.94$ & $36.00 \pm 9.52$ & $0.02 \pm 4.43$ \\
\hline $\operatorname{RMR}\left(\mathrm{kcal} \mathrm{d}^{-1}\right)$ & $1460.3 \pm 219.1$ & $102.9 \pm 187.7$ & $1629.4 \pm 356.9$ & $19.8 \pm 266.3$ \\
\hline RER & $0.78 \pm 0.06$ & $0.01 \pm 0.10$ & $0.77 \pm 0.06$ & $0.04 \pm 0.07$ \\
\hline \multicolumn{5}{|c|}{ † significant interaction (intensity * time) $\mathrm{p}<0.05$; High Density Lipoprotein, HDL; Low Density } \\
\hline Lipoprotein, LDL; Triglyceride & TG; Resting Metabo & Rate, RMR; Respir & ory Exchange Ratio, RER & \\
\hline
\end{tabular}

541

542

543

544

545

546 
Table 5: Appetite hormones and HOMA 2 parameters at baseline and after 8 weeks exercise training

\begin{tabular}{|c|c|c|c|c|}
\hline & \multicolumn{2}{|c|}{ OV/OB $(n=23)$} & \multicolumn{2}{|c|}{$L(n=11)$} \\
\hline & Pre & Post & Pre & Post \\
\hline $\begin{array}{l}\text { Fasting Leptin (ng } \mathrm{ml}^{-} \\
\text {1) }\end{array}$ & $36.25 \pm 15.76 *$ & $36.38 \pm 16.40 *$ & $16.00 \pm 11.90$ & $14.83 \pm 12.52$ \\
\hline $\begin{array}{l}\text { Fasting Insulin ( } \mathrm{mU} \mathrm{I}^{-} \\
{ }^{1} \text { ) }\end{array}$ & $7.52 \pm 3.19 *$ & $7.93 \pm 3.69 *$ & $4.34 \pm 1.93$ & $4.84 \pm 2.61$ \\
\hline $\begin{array}{l}\text { Postprandial Insulin } \\
\left(\mathrm{mU} \mathrm{I}^{-1}\right) \\
\text { Postprandial Insulin }\end{array}$ & $40.99 \pm 19.43 *$ & $47.48 \pm 19.45 *$ & $31.05 \pm 17.35$ & $38.15 \pm 23.50$ \\
\hline Change $\left(\mathrm{mU} \mathrm{l}^{-1}\right)$ & $33.46 \pm 19.21$ & $39.55 \pm 19.41$ & $26.27 \pm 17.95$ & $33.47 \pm 23.59$ \\
\hline $\begin{array}{l}\text { Fasting Amylin (pg ml- } \\
{ }^{1} \text { ) }\end{array}$ & $16.16 \pm 3.85 *$ & $12.25 \pm 3.33 \#$ & $11.96 \pm 6.20$ & $11.66 \pm 5.27$ \\
\hline $\begin{array}{l}\text { Postprandial Amylin } \\
\left(\mathrm{pmol} \mathrm{I}^{-1} \text { ) }\right. \\
\text { Postprandial Amylin }\end{array}$ & $20.42 \pm 3.64$ & $17.55 \pm 3.96 \#$ & $18.77 \pm 7.89$ & $20.96 \pm 7.74$ \\
\hline Change $\left(\mathrm{pmol}^{-1}\right)$ & $4.26 \pm 2.76 *$ & $5.35 \pm 4.45 *$ & $8.27 \pm 4.69$ & $9.64 \pm 6.82$ \\
\hline $\begin{array}{l}\text { Fasting Ghrelin (pg } \\
\mathrm{ml}^{-1} \text { ) }\end{array}$ & $677.0 \pm 254.3$ & $674.5 \pm 244.8$ & $797.4 \pm 259.2$ & $823.4 \pm 321.7$ \\
\hline $\begin{array}{l}\text { Postprandial Ghrelin } \\
\left(\mathrm{pg} \mathrm{m}^{-1} \text { ) }\right. \\
\text { Postprandial Ghrelin }\end{array}$ & $452.3 \pm 205.0$ & $491.5 \pm 216.4 \#$ & $566.3 \pm 200.2$ & $524.1 \pm 179.2$ \\
\hline Change (pg ml $\mathrm{l}^{-1}$ ) & $-224.71 \pm 126.72$ & $-183.00 \pm 93.74 \#$ & $-231.13 \pm 87.67$ & $-299.32 \pm 162.07$ \\
\hline Fasting PYY (ng/ml) & $146.85 \pm 53.17$ & $147.60 \pm 64.92$ & $118.70 \pm 60.71$ & $133.35 \pm 50.89$ \\
\hline $\begin{array}{l}\text { Postprandial PYY } \\
\text { (ng/ml) } \\
\text { Postprandial PYY }\end{array}$ & $206.73 \pm 63.29$ & $243.56 \pm 54.90$ & $227.38 \pm 75.74$ & $241.78 \pm 81.36$ \\
\hline Change (ng/ml) & $62.87 \pm 65.70^{*}$ & $95.96 \pm 53.72$ & $108.68 \pm 40.30$ & $108.43 \pm 46.10$ \\
\hline Beta Cell Function (\%) & $111.34 \pm 31.14 *$ & $109.91 \pm 30.37$ * & $76.73 \pm 22.90$ & $73.33 \pm 26.67$ \\
\hline Insulin Sensitivity (\%) & $125.19 \pm 55.43 *$ & $118.19 \pm 48.85 *$ & $221.09 \pm 113.02$ & $200.09 \pm 91.49$ \\
\hline Insulin Resistance (IR) & $0.95 \pm 0.41 *$ & $1.02 \pm 0.48 *$ & $0.55 \pm 0.25$ & $0.62 \pm 0.34$ \\
\hline
\end{tabular}

548

Significant $(p<0.05)$ effects of group, $*$, interaction (group $x$ time), \#

549

550

551

552

553

554

555 
556 Figure 1: Individual changes in body characteristics in control and exercise group after 4 weeks

557 exercise training. Black bars depict changes of overweight/obese individuals and empty bars of lean 558 individuals.

559

560

561

562

563

564

565

566

567

568

569

570

571

572

573

574

575 
576 Figure 2: Individual changes in body characteristics after 8 weeks exercise training. Black bars depict

577 changes of overweight/obese (OV/OB) individuals and empty bars of lean individuals (L). 
Control $^{\text {Applied Physiology, Nutrition, and Metabolism }}$
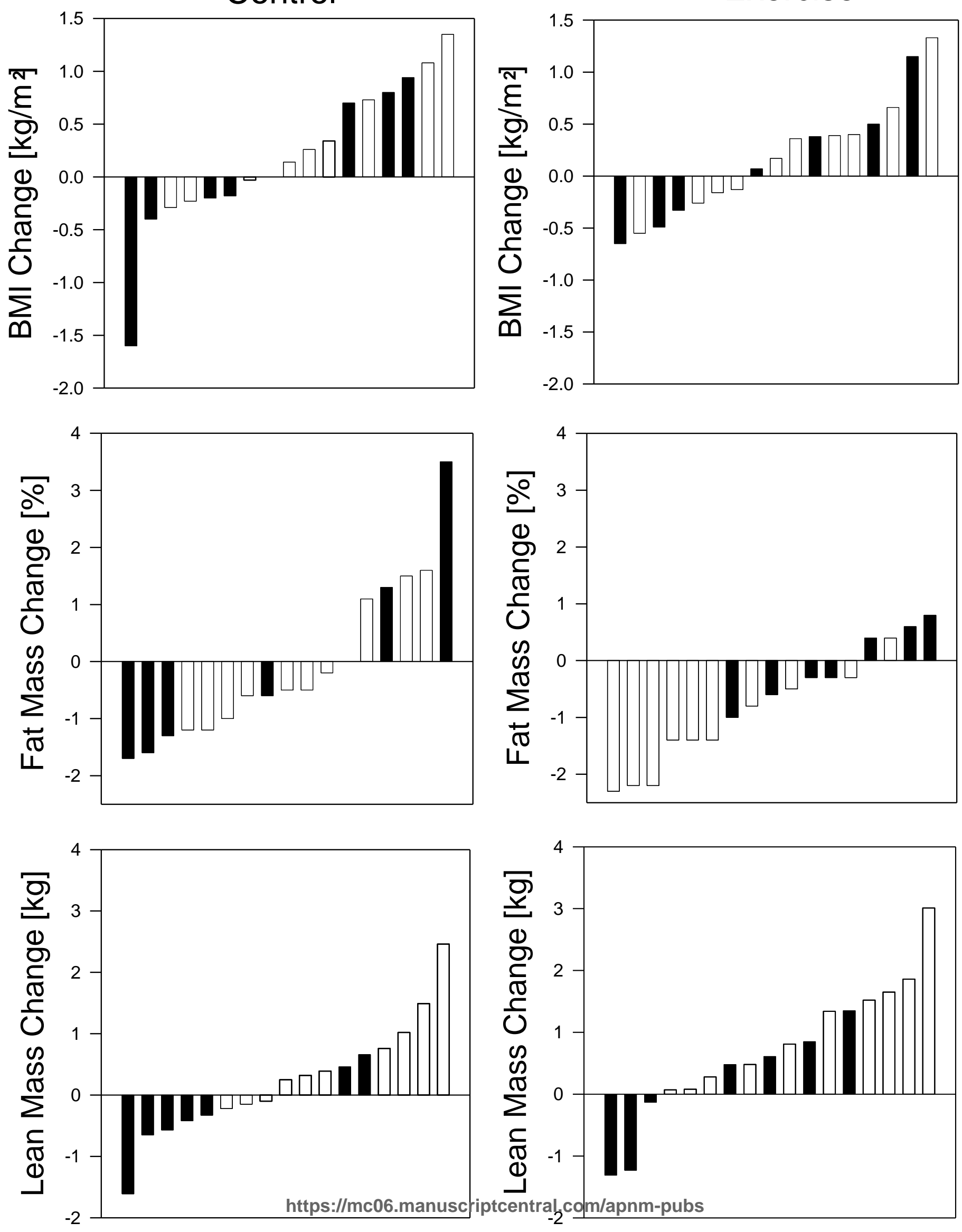


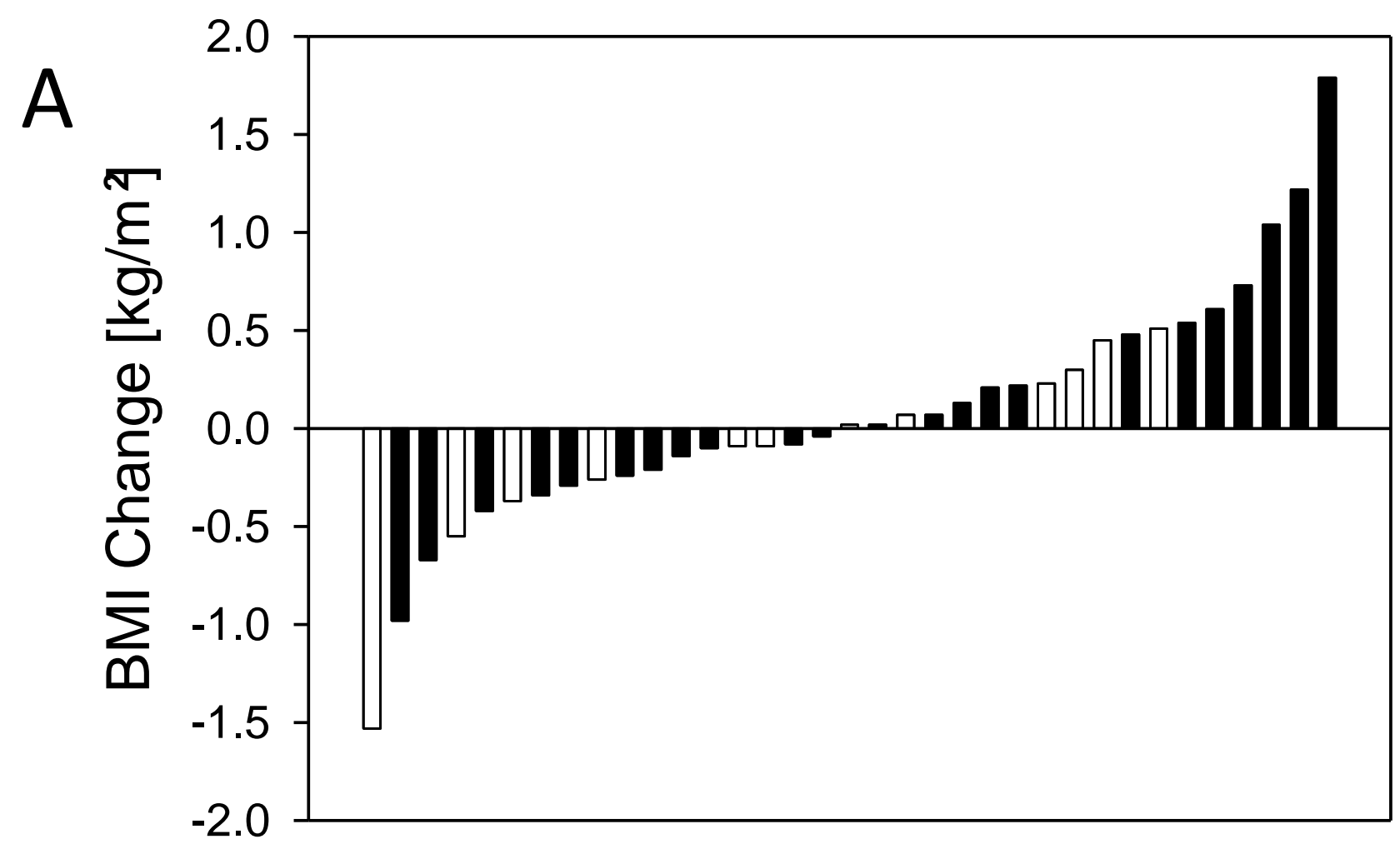

B

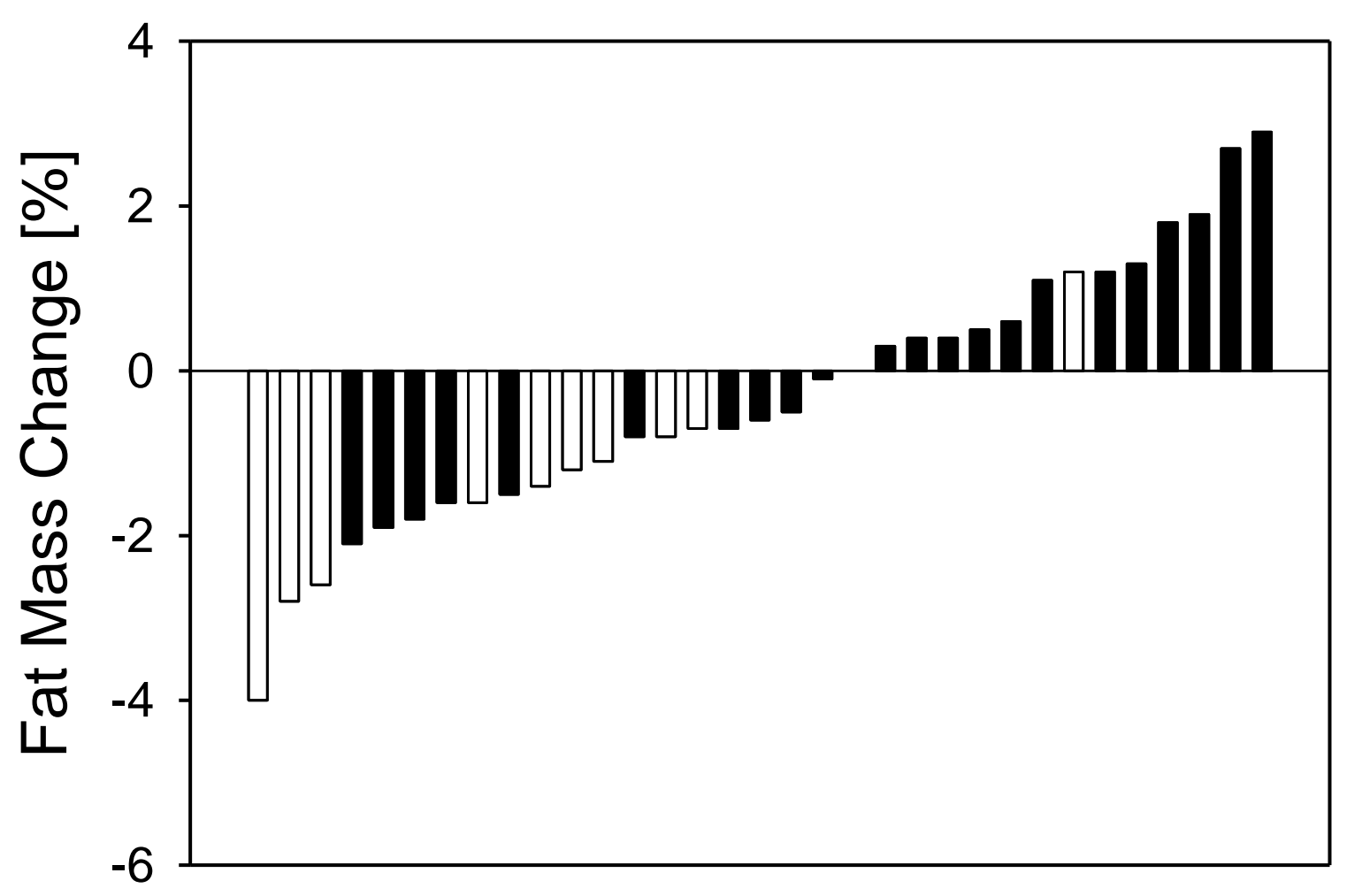

C

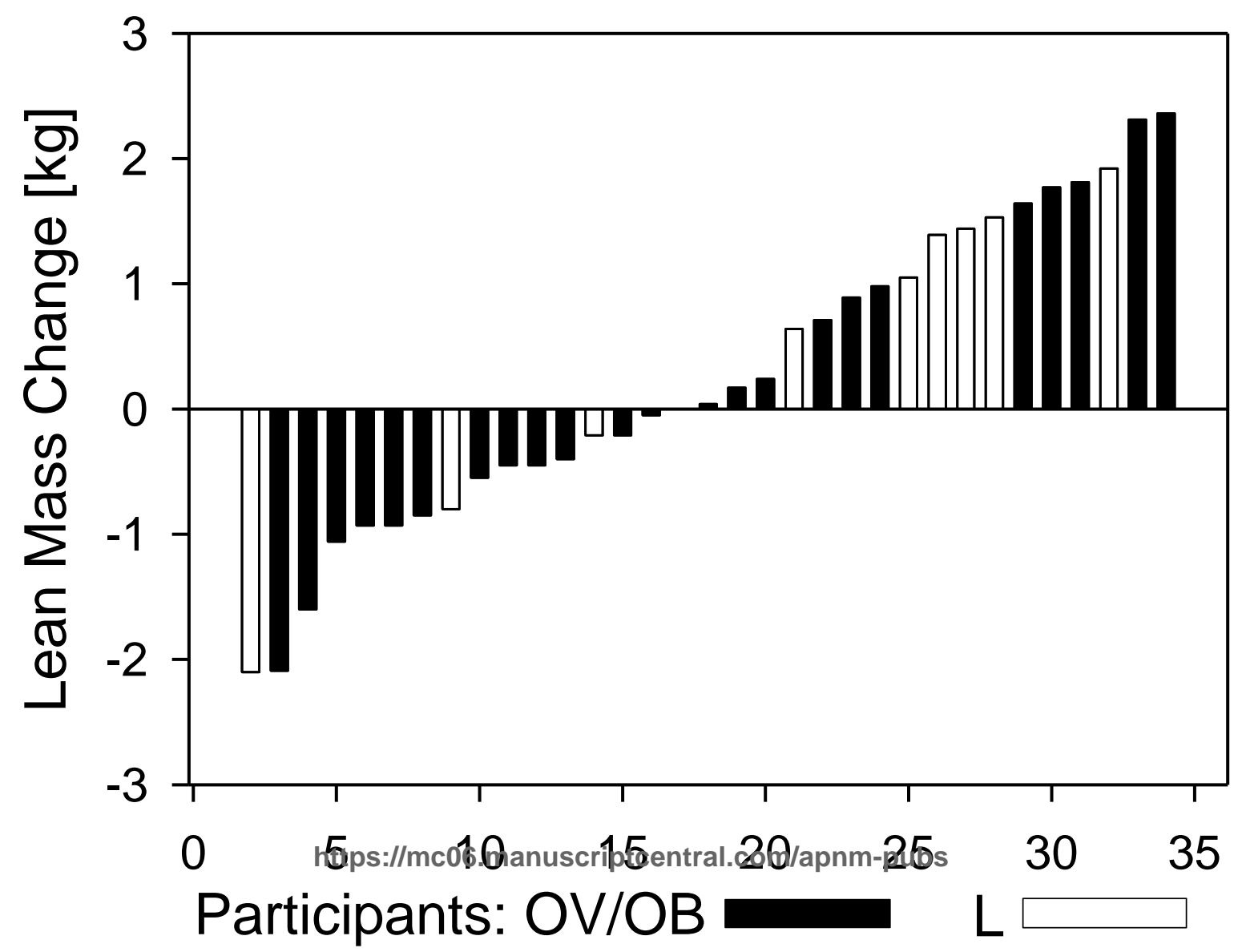

